\title{
Haemodynamic bradycardia in tachycardiomyopathy
}

\author{
M. Mafirad • Y. Blaauw • J. M. van Opstal • \\ H. J. G. M. Crijns
}

Published online: 17 May 2011

(C) The Author(s) 2011. This article is published with open access at Springerlink.com

A 44-year-old man presented with a 6-month history of progressive dyspnoea (NYHA class III). The electrocardiogram showed sinus rhythm with continuous short runs of atrial tachycardia resulting in a heart rate of 200 beats/min (Fig. 1a). An echocardiogram demonstrated left ventricular dilatation and a reduced ejection fraction of $15-25 \%$. During runs of atrial tachycardia, the mitral valve failed to open due to high end-diastolic pressures, resulting in impaired filling of the left ventricle (Fig. 1b). As a consequence there was a virtual absence of blood flow through the aortic valve during tachycardic runs
(Fig. 1c). Effective cardiac output was only seen during sinus beats, which came at a rate of $25-30$ beats per minute. The patient was diagnosed with tachycardiomyopathy due to incessant atrial tachycardia. Cryoablation of the atrial focus eliminated the tachycardia. Three months later, tachycardias were still absent and the patient's left ventricular systolic function and clinical condition had markedly improved.

Tachycardiomyopathy is an abnormality of systolic and/or diastolic function of the heart, resulting in atrial and ventricular dilatation and ultimately development of heart failure symp-

M. Mafirad $(\bowtie) \cdot$ Y. Blaauw $\cdot$ J. M. van Opstal $\cdot$

H. J. G. M. Crijns

Department of cardiology, Maastricht University Medical Centre,

P. Debeyelaan 25,

6229 HX, Maastricht, the Netherlands

e-mail:m.mafirad@alumni.unimaas.nl 

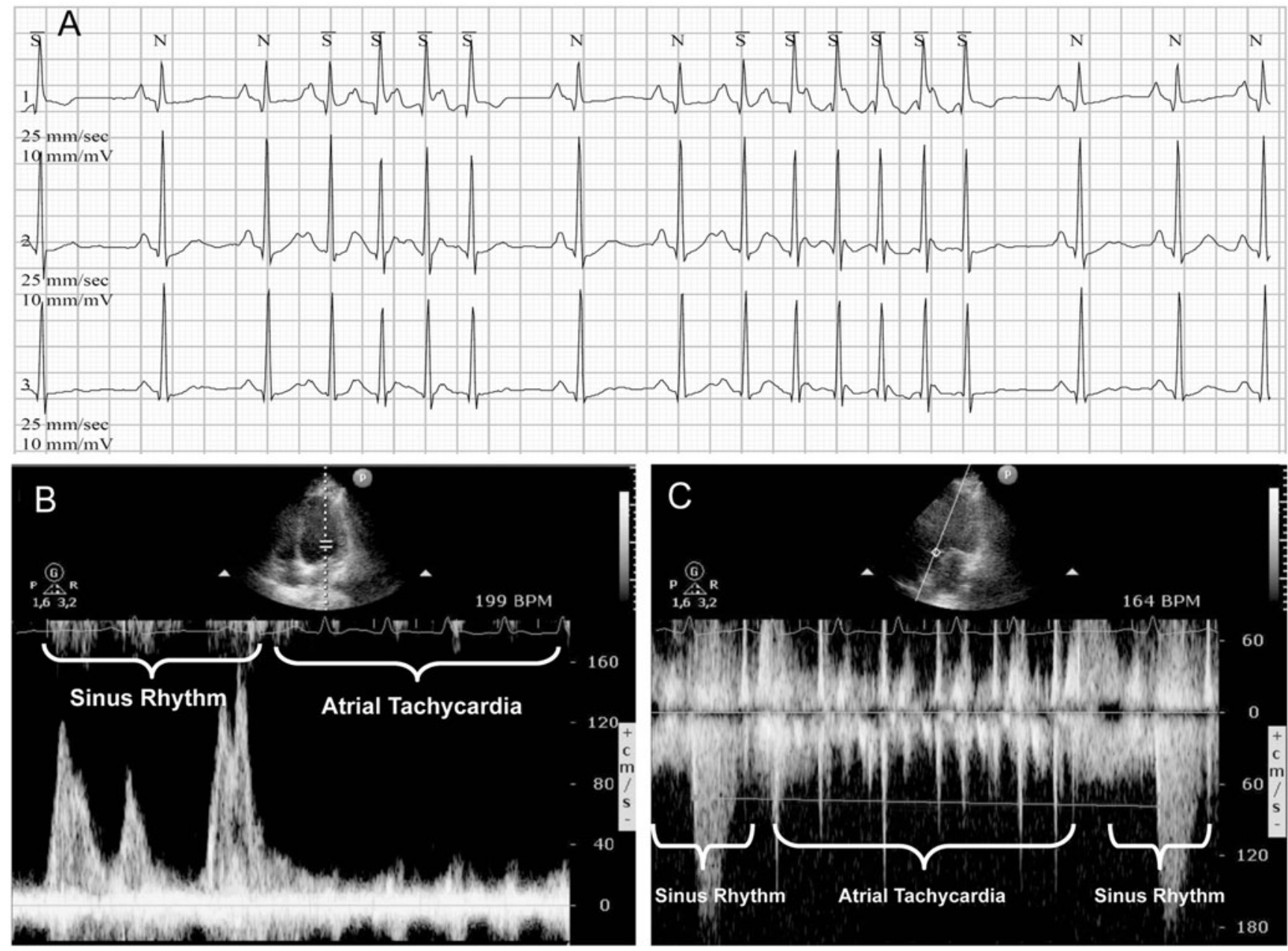

Fig. 1 a Rhythm strip demonstrating sinus rhythm and episodes of atrial tachycardia. b and c) Echocardiogram with apical views demonstrating absence of flow through the mitral valve and aortic valve during a run of atrial tachycardia

toms. Tachycardiomyopathy is usually caused by atrial arrhythmias that results in a high and/or irregular ventricular rate. Myocardial dysfunction is completely or partially reversible after normalisation of heart rate and/or rhythm irregularity [1]. The mechanisms responsible for the contractile dysfunction and structural changes in tachycardiomyopathy are not yet fully understood. This case illustrates the direct effect of tachyarrhythmia on cardiac function and its haemodynamic consequences in a patient with tachycardiomyopathy.
Open Access This article is distributed under the terms of the Creative Commons Attribution Noncommercial License which permits any noncommercial use, distribution, and reproduction in any medium, provided the original author(s) and source are credited.

\section{References}

1. Brugada P, Andries E. "Tachycardiomyopathy". The most frequently unrecognized cause of heart failure? Acta Cardiol. 1993;2:165-9. 